\title{
Experimental calibration of the ARA radio neutrino telescope with an electron beam in ice
}

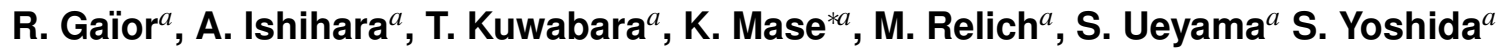 \\ for the ARA collaboration, \\ M. Fukushima ${ }^{b}$, D. Ikeda ${ }^{b}$, J. N. Matthews ${ }^{c}$, H. Sagawa ${ }^{b}$, T. Shibata ${ }^{d}$, B. K. Shin and $^{e}$ \\ G. B. Thomson ${ }^{c}$
}

${ }^{a}$ Department of Physics, Chiba University, Yayoi-cho 1-33, Inage-ku, Chiba 263-8522, Japan

${ }^{b}$ ICRR, University of Tokyo, 5-1-5 Kashiwanoha, Kashiwa, Chiba 277-8522, Japan

${ }^{c}$ Physics And Astronomy, University of Utah, 201 South, Salt Lake City, UT 84112, U.S.

${ }^{d}$ High Energy Accelerator Research Organization (KEK), 2-4 Shirakata-Shirane, Tokai-mura, Naka-gun, Ibaraki, 319-1195, Japan

${ }^{e}$ Department of Physics, Hanyang University, 222 Wangsimni-ro Seongdung-gu Seoul, 133-791, Korea

${ }^{*}$ E-mail: masedhepburn.s.chiba-u.ac.jp

Askaryan Radio Array (ARA) is being built at the South Pole, aiming to observe high energy cosmogenic neutrinos above $10 \mathrm{PeV}$. The ARA detector aims to observe the radio emissions from the excess charge in a particle shower induced by a neutrino interaction. Such a radio emission was first predicted by Askaryan in 1962 and experimentally confirmed by Saltzberg et al. using the SLAC accelerator in 2000. We also performed an experiment of the ARA calibration with the Telescope Array Electron Light Source (ARAcalTA) to verify the understanding of the Askaryan radiation and the detector response used in the ARA experiment. In the ARAcalTA experiment, we irradiated an ice target with $40 \mathrm{MeV}$ electron beams using the Telescope Array Electron Light Source (TA ELS) located in a radio quiet open-air environment of the Utah desert. Observed signals would include two kind of backgrounds: transition radiations from the boundary between air and ice, and radio emissions from the sudden beam appearance. We measured coherences, polarizations and angular distributions of the radio signals to understand the observed signals. These first observational results from ARAcalTA are presented in these proceedings.

The 34th International Cosmic Ray Conference,

30 July- 6 August, 2015

The Hague, The Netherlands

\footnotetext{
* Speaker.
} 


\section{Introduction}

The origin of ultra high energy cosmic rays (UHECRs) with the energies above $5 \times 10^{19} \mathrm{eV}$ has not yet been revealed completely, although ground-base large detectors such as Telescope Array (TA) and Auger have been observing UHECRs more precisely with large statistics. UHECRs interact with cosmic microwave background photons during their propagation to the Earth and

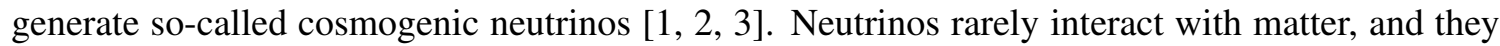
come from the deep universe, so the neutrino flux we observe on the Earth reflects the history of cosmic-ray source evolution from the beginning of the Universe up to now. Therefore, the observation of cosmogenic neutrinos can elucidate the origin of UHECRs.

IceCube has performed a search for cosmogenic neutrinos with energies above $100 \mathrm{PeV}$ by observing Cherenkov light derived from secondary relativistic charged particles initiated by neutrino interactions [䧃]. Although the expected event rate depends on the evolution models of cosmic-ray sources and the composition, the rate is about one event per year in the optimistic case of a proton composition. IceCube has not yet observed cosmogenic neutrinos. The Askaryan Radio Array (ARA) detectors are being built at the South Pole to extend the ability to search for cosmogenic neutrinos by about one order of magnitude [ [5].

The ARA detector aims to observe coherent radio emissions from a net negative charge excess produced in a cascade shower after a neutrino interaction. This was first predicted by G. A. Askaryan [ [G] and this Askaryan effect was confirmed experimentally by D. Saltzberg et al. at SLAC [ $[\mathbb{\text { U }}]$. Since the power of Askaryan radiation is quadratically proportional to the amount of the charge excess in an electro-magnetic (EM) shower, the Askaryan radiation becomes prominent at energies above $10 \mathrm{PeV}$. In addition, the attenuation length in the ice for radio waves with the frequencies of our interest (200-800 MHz) is about one kilometer, and longer than the one for visible light (about $100 \mathrm{~m}$ ). Therefore, the Askaryan radiation is the optimal tool to search for cosmogenic neutrinos.

The understanding of Askaryan radiation as well as the detectors is crucial for the ARA experiment. In order to verify the understanding of Askaryan radiation and the ARA detector units, we performed an experiment of the ARA calibration with the TA Electron Light Source [8] (ARAcalTA).

\section{The ARAcalTA experiment}

We performed the ARAcalTA experiment using the TA-ELS $40 \mathrm{MeV}$ electron beams at the TA site in the middle of the desert in Utah in January, 2015. In order to confirm the Askaryan radiation, several features had to be measured. One important feature is coherence of the radiation. The radiation power is expected to be quadratically proportional to the electron number in an accelerator beam bunch train. Another feature is the angular distribution. A wide Gaussian-like distribution peaking around almost horizontal direction with regard to the beam direction is expected. This is because the interference condition is weak due to the short electron track compared to the wavelength of observation [Q]. The last feature is the polarization. The radiation is expected to be highly polarized in a radial direction with regard to the beam axis. The ARAcalTA experiment was con- 

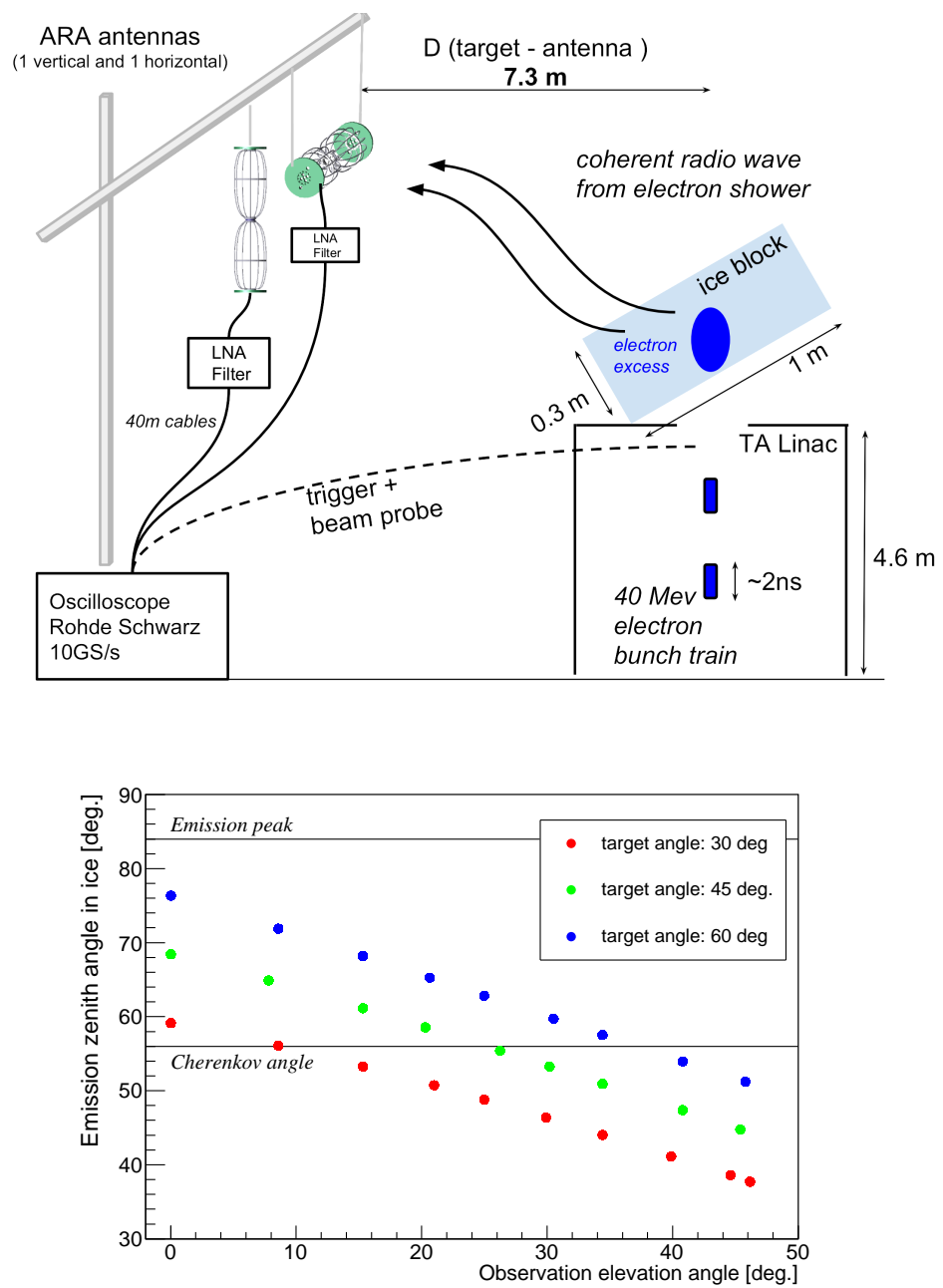

Figure 1: Top: a schematic of the ARAcalTA experiment. Bottom: the relation between the emission zenith angle in ice and the observation elevation angle from the target to the antenna by taking the refractive index of the ice $(n=1.78)$ into account. Each point represents an observation elevation angle of the antenna when an actual measurement was performed. The elevation angle is limited by the maximum antenna tower height. Each ice target inclination angle covers a different emission angle range, so that a wide emission angle range is covered by changing the target inclination angle. Note also that the peak of the angular distribution for the expected Askaryan radiation is shifted from the Cherenkov angle $\left(56^{\circ}\right)$ due to the short electron track length [Q].

figured as shown in Fig. $\square$ to measure these Askaryan radiation features, intending to recreate the actual condition in the ARA experiment as close as possible.

The $40 \mathrm{MeV}$ electron beam from TA-ELS consists of bunch trains that includes about 10 bunches with a time separation of 350 ps. The duration time of a bunch train was configured to $1.9 \mathrm{~ns}$ (FWHM) which was changed from the TA original of $1 \mu$ s to enhance a signal amplitude as much as possible by avoiding interference caused by the bunch structure. The rate of the beam was $0.5 \mathrm{~Hz}$ and the maximum electron number in a bunch train is limited to $10^{9}$ by a radiation safety regulation. The electron number was changed during measurements to confirm the coherence of 
radiations by controlling the supply voltage for the accelerator heater. The total electron number was directly measured by a Faraday Cup (FC). Since the FC absorbs all the electrons, it can not be used when electron beams are shot to the target. A Wall Current Monitor (WCM), which measures the current on the beam pipe induced by the beam, was used to estimate the electron number while radio emissions were observed. A clear correlation between the charge from the FC and the one from the WCM was found, and the uncertainty of the electron number was estimated to be $0.2 \%$.

The electron beam was injected to an ice target with the size of $100 \mathrm{~cm} \times 30 \mathrm{~cm} \times 30 \mathrm{~cm}$ placed above the beam end pipe. The lateral beam spread at the injection point is estimated to be $4.5 \mathrm{~cm}$ $(1 \sigma)$ by GEANT4 simulation. The ice target was supported by a plastic box that has a slit at the bottom with the width of $25 \mathrm{~cm}$ to directly inject beams to the target.

Electrons run about $20 \mathrm{~cm}$ and stop inside the target, simply losing the energy by ionizations. Those electrons are used to emulate a high energy EM shower in an ice target that will generate Askaryan radiation, even though a cascade is not generated in ice since the electron energy is lower than the critical energy of $69 \mathrm{MeV}$ in ice. The corresponding energy for an EM shower can be estimated by the energy of each electron, the total number of electrons in a bunch train and a negative charge excess in a shower which is about $25 \%$. In the ARAcalTA experiment, the electron energy is $40 \mathrm{MeV}$ and the typical electron number is about $2 \times 10^{8}$, so, the corresponding EM shower energy is about $30 \mathrm{PeV}$. This energy is high enough to produce Askaryan radiation, therefore, the radiation can be observed in the ARAcalTA experiment.

The radio emissions were observed by the ARA antennas installed on top of an antenna tower using an antenna mast. The tower was fixed to the ground with the horizontal distance of $7.3 \mathrm{~m}$ away from the beam injection point to satisfy the far-field condition. Two ARA bicone antennas were used and placed horizontally and vertically, being hung from the mast, to measure the polarizations of radio emissions with the separation of $126 \mathrm{~cm}$ between two centers. The observed signals were then filtered by band-pass filters (230-430 MHz) and amplified by low noise amplifiers with the gain of $35 \mathrm{~dB}$. This filter is not used for ARA but only for the ARAcalTA experiment to filter environmental noise at the TA site. The signals were transferred with $40 \mathrm{~m}$ cables to a fast oscilloscope $(10 \mathrm{GSa} / \mathrm{s})$ to be digitized. The height of the antennas could be changed by the antenna tower up to $12.2 \mathrm{~m}$ from the ground. Radio emissions were measured about every $1 \mathrm{~m}$ to probe the angular distribution of the Askaryan radiation. Since the height of the beam injection point is $4.6 \mathrm{~m}$, the accessible elevation angle from the target to the antenna is limited up to $45^{\circ}$. The ice target was inclined to angles of $30^{\circ}, 45^{\circ}$ and $60^{\circ}$ to access different emission angles in ice as shown in Fig. 四.

We performed the experiment over two weeks using the accelerator in 8 days in total. The measurements were performed during the day not to disturb the TA fluorescence observation. We took data for each configuration of the ice inclination angle and each height as well as the one without any target. Each measurement contains typically 200 events.

\section{Expected Askaryan radiation and backgrounds}

Askaryan radiation is expected to be produced by the electron beam inside the ice target in the ARAcalTA experiment. The radiation is expected to show coherence, a high polarization and a wide angular distribution for our experiment. The expected waveforms and the signal powers 
were simulated for each measurement configuration of different elevation angle of the antenna and the ice inclination angle using the actual accelerator configurations. These results are presented in these proceedings [9], showing the Askaryan radiation is expected to be larger than thermal noises. The simulated waveform and the frequency spectrum are shown in Fig. \.

Two other backgrounds would also be included for our experiment. The first background could be transition radiation (TR) due to the transition from air to the ice target, which have been significant in previous experiments [U]]. We would also expect significant TR in this experiment based on the short track length in the ice block compared to the frequencies of interest [ए]]. The second background would be radiation due to a sudden appearance of the electron beam, similar to the expected coherent radio emission from the cosmic ray air shower sudden death [एँ2]. The radio signals from the sudden appearance was experimentally measured by removing the ice target from the beam line, however, the contribution from the sudden appearance would be different when we used the ice target and the electrons were stopped inside the ice.

\section{Results}

Clear signals of radio emissions were observed especially for the vertically placed ARA bicone (Vpol) antenna for all the configurations with the ice target. Smaller signals were observed for the horizontally placed (Hpol) antenna. The waveforms of Vpol and Hpol antenna information already show the high polarizations of observed radio emissions. An example of a waveform averaged over the 200 events is shown in Fig. $\square$ for a configuration of the ice inclination angle of $30^{\circ}$ and the antenna elevation angle of $0^{\circ}$. The corresponding simulated waveform is compared with the data. The large difference of the amplitude between data and simulation was found. The observed signal amplitude is about 6 times larger than the expected signals from Askaryan radiation, which indicates another emission. The waveform shapes between data and simulation are similar for the first part, though there is a difference for the later part, which also indicates another background component. The frequency spectra for each waveform are also shown in Fig. \, where the effect due to the band-pass filter to filter environmental noise is clearly seen. The dominant contribution is expected to come from the lowest frequencies around $230 \mathrm{MHz}$. This is different from the standard case of the Askaryan radiation, where more contribution is expected from higher frequency signals. This is due to the bunch structure of the accelerator beam and our detector responses [Q].

Several data were taken with same configurations to check the reproducibility. The observed signals were found to be stable, the waveform having the same shape. The stability of the maximum signal amplitude of the averaged waveforms was found to be less than 5\%. The stability of the observed signal time from the start time of the beam was also found to be less than $100 \mathrm{ps}$. In order to further assess the stability of the system, the antenna tower was intentionally rotated in azimuthal angle by $15^{\circ}$. The waveform shapes changed slightly for the Hpol antenna due to the angular dependence of the antenna gain, which is correctable taking the gain into account. On the other hand, the shape was unchanged for the Vpol antenna because the antenna kept the same direction to the target after the rotation. The waveform amplitude decreased by $11 \%$ after the rotation. The amount of the decrease was proportional to the geometrical distance change calculated with the signal time delay. This result confirms that our antennas was at far-field region. The delay time was used to monitor the direction of the antenna mast during the measurements. The spread 

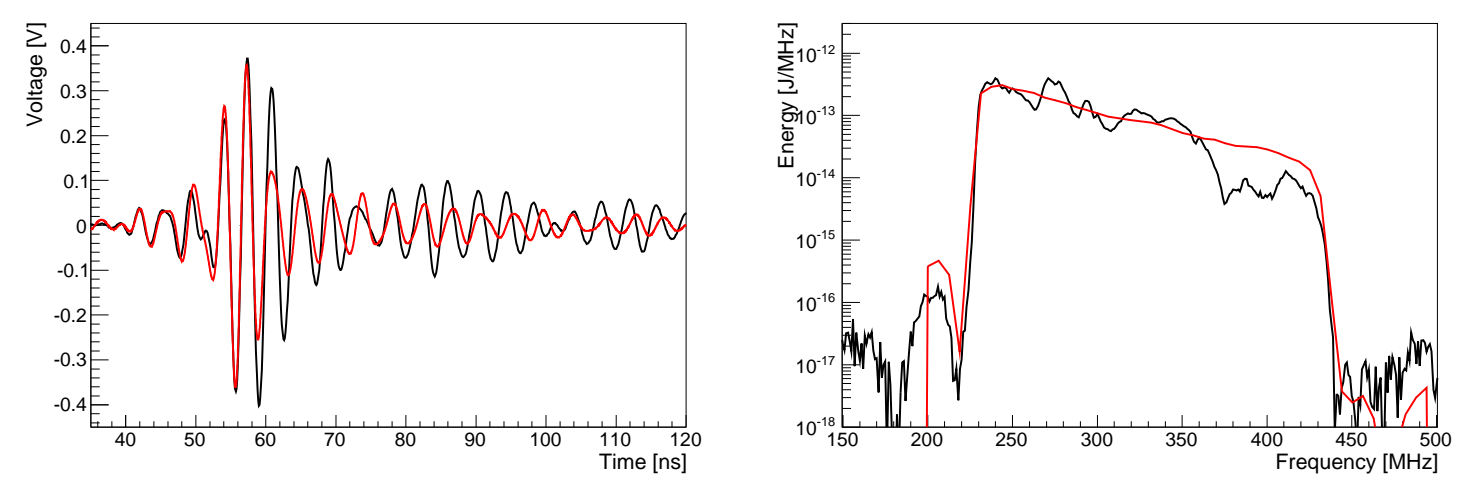

Figure 2: Left: Averaged waveform measured by the vertically polarized antenna with a configuration of the ice inclination angle of $30^{\circ}$ and the antenna elevation angle of $0^{\circ}$ (black) and a simulated corresponding waveform for the Askaryan radiation normalized to the observed waveform (red). Right: The corresponding frequency spectrum. The sharp cutoffs are due to the band-pass filter to filter environmental noise.

(rms) of the delay time from the expectation was found out to be $1.9 \mathrm{~ns}$. This corresponds to a $9^{\circ}$ rotation angle and the distance change by the rotation will change the waveform amplitude by $6 \%$. The uncertainty of the antenna power gain was estimated based on data measured in an anechoic chamber, and it is $5-17 \%$ depending on the angle $\left(0^{\circ}-45^{\circ}\right)$. The overall systematic uncertainties on the measured radio energy was estimated based on those measurements and it is $17-23 \%$ depending on the angle.

Clear signals were also observed even without any target. The signal amplitude is about one third of the signals with the ice target at an antenna elevation angle of $0^{\circ}$. The emission is thought to be due to a sudden appearance of the beam electron charge. This emission is thought to be also observed with the ice target and would be the background for our measurements.

The polarizations of signals were first investigated using the Vpol and Hpol antenna signal information. High vertical polarizations of $0.92 \pm 0.03$ were found in all observed signals with the ice target. The polarization for the case without the target was also checked, and a lower polarization value of $0.82 \pm 0.03$ was found. The distribution of the polarization and the angle are shown in Fig. [1].

The coherence of the measured signals were next investigated to understand the property of the measured signals. The signal power is calculated by integrating the square of the waveform amplitude for all the waveform. All observed signals, both for Vpol and Hpol, with any configuration show a high coherence. The relation between the electron number and radio power for one run is shown in Fig. . The high coherence was also found even in the case of no target. The slope index for coherence is derived as $1.78 \pm 0.07$ from all the configurations with Vpol antenna.

To investigate the angular distribution of measured emissions, the measured radio emission energies are plotted as a function of the elevation angle of the antenna for each ice inclination angle as shown in Fig. [1. The expected energies from Askaryan radiation calculated in a way in Ref. [Q] are also plotted for each configuration. As seen from the plot, we observed more than one order of magnitude larger emissions compared to expected Askaryan radiation. 

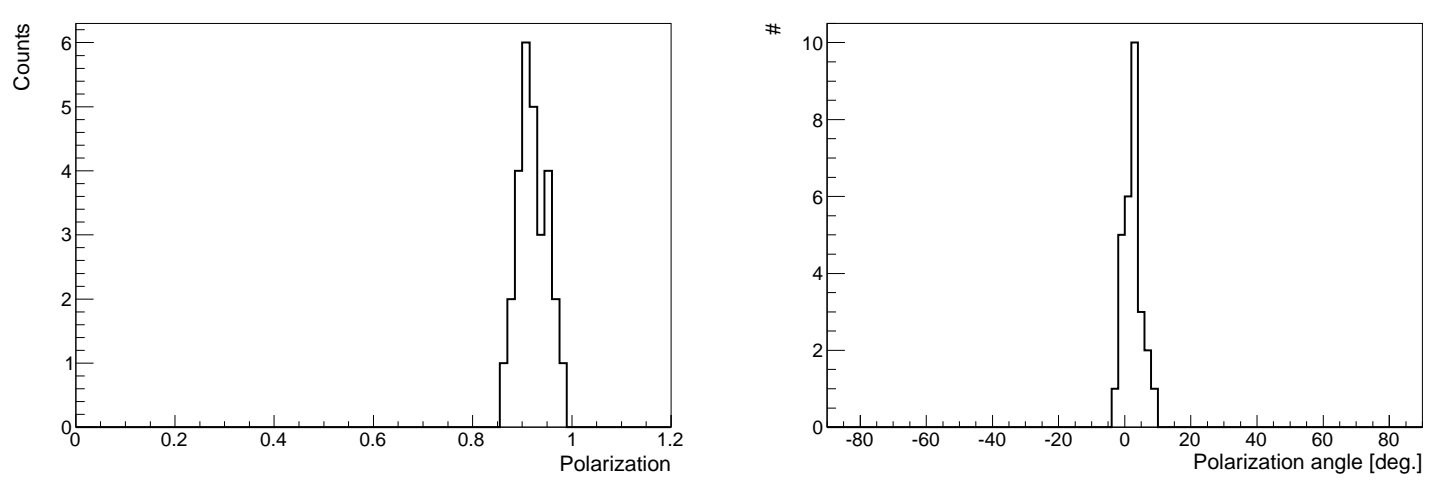

Figure 3: Left: Polarization of measured radio emissions for all the configurations with the ice target. Right: the polarization angle. These results show a high vertical polarization.

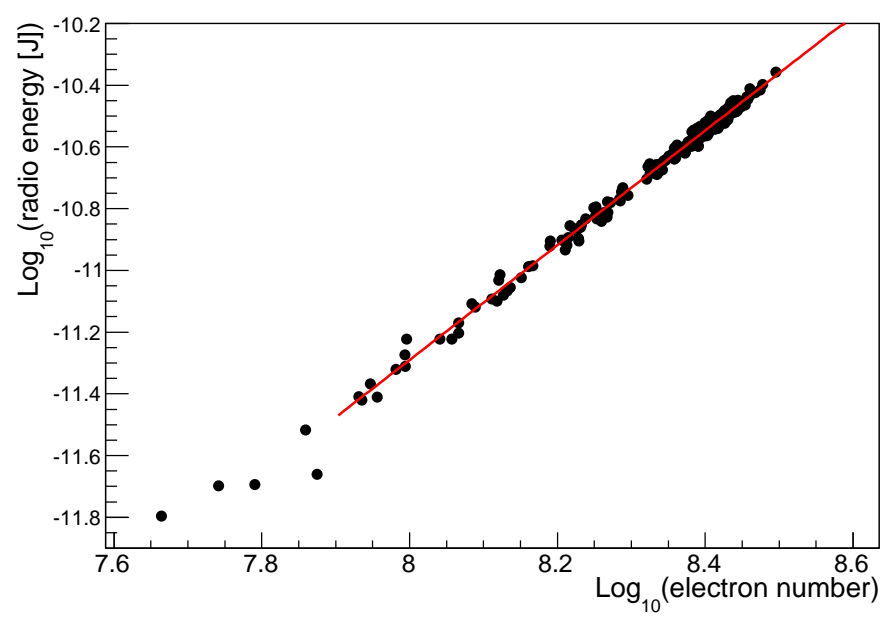

Figure 4: Measured radio emission power as a function of electron number in the beam for a configuration of the ice inclination angle of $30^{\circ}$ and the antenna elevation angle of $0^{\circ}$. The slope is $1.86 \pm 0.01$, showing a high coherence.

\section{Summary}

We have clearly observed radio signals that show high coherence and high vertical polarization from the ice target with the TA-ELS electron beam. The observed waveform amplitude is about 6 times larger than the expectation for the Askaryan radiation. We have also observed relatively large radio signals from the beam itself. Since this sudden appearance background does not account for all the measured radio signals, the observed signals would be very likely to contain TR. Our current focus is to explain the excess by a full simulation which takes into account all the backgrounds and the Askaryan signals. The mechanism of the Askaryan radiation and TR is closely related, and it is difficult to separate those two radiations. These radiations could also be reduced because the length of the electron track in the target is comparable to the wavelength of our interest. To estimate these inseparable radiations, we are developing a simulation based on the endpoints method described in 


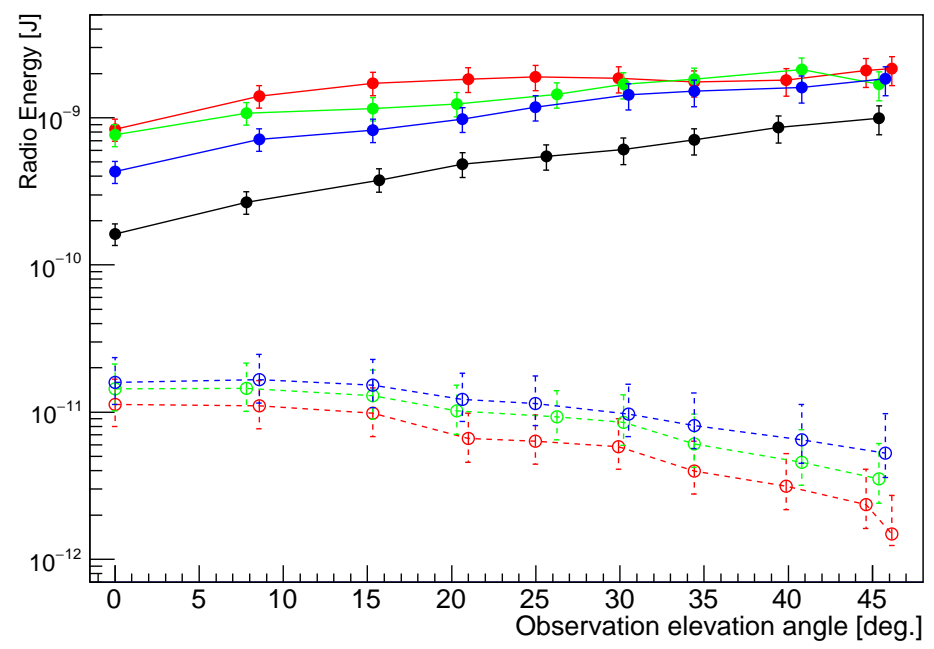

Figure 5: Observed radio emission energy as a function of the observation elevation angle of the antenna for the cases with ice target of the inclination angles of $30^{\circ}$ (red filled circle), $45^{\circ}$ (green filled circle), $60^{\circ}$ (blue filled circle) and without any target (black filled circle). The measured energy is normalized to the typical electron number of $2 \times 10^{8}$. Note that the antenna gain is corrected and the energy is normalized to the one at $1 \mathrm{~m}$ from the source to correct the geometrical factor. The expected radio emission energy from Askaryan radiation from the same amount of electron numbers for each configuration is also plotted with the open circle (The color represents the ice inclination angle as same as the measurements).

Ref. [ए3] which treats all radiations in a consistent way.

\section{References}

[1] V. Berezinsky and G. Zatsepin, Phys. Lett. 28B, 423 (1969).

[2] S. Yoshida and M. Teshima, Prog. Theor. Phys. 89, 833 (1993).

[3] M. Ahlers, L. A. Anchordoqui, and S. Sarkar, Phys. Rev. D 79, 083009 (2009).

[4] M. G. Aartsen et al. (IceCube Collaboration), Phys. Rev. D 88, 112008 (2013).

[5] P. Allison et al. (ARA Collaboration), Astropart. Phys. 35, 457 (2012).

[6] G. A. Askaryan, JETP 14, 441 (1962).

[7] D. Saltzberg et al., Phys. Rev. Lett. 86, 13 (2000).

[8] T. Shibata et al., Nucl. Instrum. Methods Phys. Res., Sect. A 597, 61 (2008).

[9] R. Gaïor et al., PoS(ICRC2015)1135 these proceedings.

[10] P. W. Gorham et al., Phys. Rev. E 62, 6 (2000).

[11] J. Zheng et al., Physics of Plasmas 12, 093105 (2005).

[12] B. Revenu and V. Marin, paper 398, ICRC 2013 proceedings.

[13] C. W. James et al., Phys. Rev. E 84, 056602 (2011). 